\title{
TRANSMISSION ELECTRON MICROSCOPY STUDY OF VERTICAL QUANTUM DOTS MOLECULES GROWN BY DROPLET EPITAXY
}

D. Hernández-Maldonado (1) ${ }^{*}$, M. Herrera (1), D. L. Sales (1), P. Alonso-González (2), Y. González (2), L. González (2), J. Pizarro (3), P. L. Galindo (3) and S. I. Molina (1)

(1) Departamento de Ciencia de los Materiales e I.M. y Q.I., Facultad de Ciencias, Universidad de Cádiz, Campus Río San Pedro, s/n, 11510 Puerto Real, Cádiz, Spain.

(2) Instituto de Microelectrónica de Madrid (CNM-CSIC), Isaac Newton 8 (PTM), 28760-Tres Cantos (Madrid) Spain.

(3) Departamento de Lenguajes y Sistemas Informáticos, CASEM, Universidad de Cádiz, Campus Río San Pedro, s/n, 11510 Puerto Real, Cádiz, Spain.

*Correspondence: david.hernandez@uca.es

Keywords:

Quantum dots molecules

Droplet epitaxy

Transmission Electron Microscopy

Semiconductors

PACS:

68.37. Lp

81.05. Ea

81.15.Hi 


\begin{abstract}
:
The compositional distribution of InAs quantum dots grown by molecular beam epitaxy on GaAs capped InAs quantum dots has been studied in this work. Upper quantum dots are nucleated preferentially on top of the quantum dots underneath, which have been nucleated by droplet epitaxy. The growth process of these nanostructures, which are usually called as quantum dots molecules', has been explained. In order to understand this growth process, the analysis of the strain has been carried out from a 3D model of the nanostructure built from Transmission Electron Microscopy images sensitive to the composition.
\end{abstract}

\title{
1. Introduction
}

Many studies have been carried out to produce site-controlled quantum dots (QD), due to the interest to introduce them into photonics crystals and memories [1, 2]. These applications require the control of size (emission wavelength) and nucleation sites of the quantum dots. In this sense, the use of droplet epitaxy growth technique [3] has the advantage of providing QD preferential nucleation sites and permits the size control of the QD [4]. The droplet epitaxy (DE) technique is a specific Molecular Beam Epitaxy (MBE) method that originally was proposed to fabricate III-V semiconductors microcrystals on II-IV substrates [3]. The method consists in the deposition of metallic III-group droplets on the surface under the absence of V-group elements. When the V-group element is introduced into the chamber, droplets crystallize into a III-V semiconductor QD. The temperature of the substrate during the crystallization permits differentiate between low temperatures $\left(200-300^{\circ} \mathrm{C}\right)$ growth process, making it possible to nucleate different nanostructures [5,6,7], and high temperatures $\left(\sim 500^{\circ} \mathrm{C}\right)$ growth process , that permits to form nanoholes on GaAs surfaces [8].

Coupling between QD in close proximity is being of great technological interest [9]. When two QD are close enough, carrier tunneling is possible between both nanostructures. A "molecule" formed by two coupled QD could be use to implement quantum gates [10]. Vertical arrangement, low density and localization of QD are needed in order to integrate these nanostructures in photonic crystals [11]. Great efforts have been done for the formation of 
vertically coupled QD [12]. In the sense of a good understanding of the growth mechanism responsible for their formation, a study of the strain and stress field is required. Different methods have been used to make strains and stress maps, being the most extended the finite element method (FEM) [13, 14].

This work is focused in the study by transmission electron microscopy techniques of low density site-located Quantum Dots Molecules (QDM) nanostructures, which consist in two vertically coupled QD grown by an optimized method, which will be explained in the next section [15]. Based on our observations and with the help of strain analysis, we propose a model of how these nanostructures have been formed.

\section{Experimental details}

The optimized experimental procedure started creating a template of nanoholes that has been fabricated by droplet epitaxy on a (001) GaAs substrate at a growth temperature of $\mathrm{T}_{\mathrm{s}}=500^{\circ} \mathrm{C}$. 1.4 monolayer (ML) of InAs were deposited on the template to form the first layer of QD (that we call QD1) at $\mathrm{T}_{\mathrm{s}}=510^{\circ} \mathrm{C}$. We have to mention that $1.7 \mathrm{ML}$ is the critical thickness to obtain a QD on a flat surface. After this, a thin intermediate layer consisting of $4 \mathrm{~nm}$ of GaAs was deposited by Atomic Layer MBE (ALMBE) growth technique at $\mathrm{T}_{\mathrm{s}}=450^{\circ} \mathrm{C}$. In the next step 0.9 ML of InAs were deposited at the same conditions used for the underlying nanostructure to nucleate a second layer of QD (QD2) above the QD1, obtaining a "molecule" structure. Finally QDM are capped by $155 \mathrm{~nm}$ of GaAs [15]. A scheme of the nanostructure is presented in Fig. 1.

Cross sectional specimens for transmission electron microscopy (TEM) were produced by standard procedures (mechanical and ion milling thinning). Electron transparency was obtained with a precision ion polishing system (PIPS) up to $3 \mathrm{KV}$ and $4^{\circ}$ of beam tilt. Images have been acquired at $120 \mathrm{KV}$ with a JEOL 1200EX transmission electron microscopy, using 002 reflection under dark field (DF) two-beam condition.

\section{Results and Discussions}


Fig.2 shows a conventional transmission electron microscopy cross-section g002 (the letter $\mathrm{g}$ denotes the Bragg reflection, each $\mathrm{g}$ is associated with an (hkl) crystal plane) DF TEM image of the studied sample. In this image it is possible to appreciate the layers that form this sample, where InAs-rich zones show darker intensity. From the analysis of similar images, we have found a low spatial density $\left(\sim 10^{8} \mathrm{~cm}^{-2}\right)$ of quantum dots molecules (QDM) and no structural defects were found (this means that if they are present their density has to be below $10^{7} \mathrm{~cm}^{-2}$ ).

Fig. 3(a) shows a 002 DF image of a QDM and wetting layers. Two well defined wetting layers separated by a GaAs layer with a nominal thickness of $4 \mathrm{~nm}$ are observed with straight horizontal dark contrasts to both sides of the QDM nanostructure. Concerning the nanostructure imaged in Fig. 3(a), several main features are observed: (i) The cleft-like shape contrast observed just below QD1 is attributed to GaAs with vacancies generated during the nanohole formation process, that are partially occupied by In and As atoms during the deposition of the first 1.4 ML of InAs. This contrast will have a combined influence of the InGaAs composition and strain. (ii) Nanohole is completely filled with an InAs rich InGaAs alloy after the deposition of 1.4ML of InAs, that present a dark contrast, forming the first layer of QD, marked as QD1 in the image. (iii) When QD as QD1 are capped with 4nm thick GaAs layer, the result is a bright mound of GaAs rich InGaAs alloy over it [4].(iv) On top of the mound we find another nanostructure, with a dark contrast and a halo around it. This corresponds with a QD of the second layer (QD2) that has been nucleated after the deposition of 0.9 ML of InAs. Indium segregation and strain effects mainly associated to QD2 are the more likely reasons explaining this halo. Location of contrasts associated to QD1 and QD2 are marked on the intensity profile of Fig. 3(b), traced across the nanostructure along the [001] direction. On the other hand, two well defined peaks appear in the intensity profile of Fig. 3 (c), that are associated to the two wetting layers separated by a thin layer nominally made up of GaAs. A deeper study, with the contribution of high-resolution Z-contrast images, is under way, and it will be published elsewhere. 
No QDs of the first layer (QD1) were found outside nanoholes, and all the analyzed QD of the second layer (QD2) were found on top of the first family of QDs'.

To understand the growth process we have used a 3D model of the QDM nanostructure, and the anisotropic elastic theory has been applied to determine by the finite element method the strain distribution during the different steps of InAs and GaAs MBE deposition. The model has been built up from TEM and Atomic Force Microscopy (AFM) [4] results. The obtained strain values reveal that when a nanohole is capped homogenously with $1.4 \mathrm{ML}$ of InAs, the nanohole and the lobes located on both sides of it (not visible in the conventional TEM images but shown in [4]) show more strain than the surrounding area, so the InAs tend to grow preferentially in those zones. After the deposition of the first layer of InAs, our TEM images show that the amount of Indium in the nanohole is larger than on the lobes, confirmed by the intensity profiles in Fig. 3(b) and 3(c) (in these images, the amount of In is related to the height of the intensity profile). Therefore, we propose that when the first 1.4ML of InAs are deposited the influence of the surface energy governs the process, that is, InAs tend to leave convex zones (the lobes), nucleating preferentially in the nanohole. When growing the intermediate layer of GaAs on top of QD1, a mound is formed. In previous studies [4] these mounds have been related with the buried strained nanostructure; the propagation of strain from QD1 to the cap layer will provide a preferential nucleation site for the growth of QD2 on top of the mounds, being the reason for quantum dots to nucleate on top of buried nanostructures.

\section{Conclusions}

TEM analysis confirm that nucleation of InAs QD, grown by MBE on buried QD formed by droplet epitaxy on (001) GaAs substrates, preferentially occurs on top of the buried nanostructures. From strain calculations, it has been deduced that the driven force for the formation of the buried QD is the surface energy, while as the strain energy controls the formation of the site-controlled upper QD grown by MBE.

\section{Acknowledgments}


This work was supported by the Spanish MCI (TEC2008-06756-C03-02/TEC) and the Junta de Andalucia (PAI research groups TEP-120 and TIC-145; project P08-TEP-03516).

\section{References}

[1] Martin-Sánchez J, Alonso-González P, Herranz J, González Y and González L Nanotechnology 20125302 (2009)

[2] Schneider C, Huggenberger A, Sünner T, Heindel T, Strau $\beta$ M, Göpfert S, Weinmann P, Reitzenstein S, Worschech L, Kamp M, Höfling S and Forchel A Nanotechnology 20434012 (2009)

[3] Koguchi N and Ishige K, Jpn. J. Appl. Phys. 322052 (1993)

[4] Alonso-González P, Fuster D, González L and Martínez-Sánchez J and González Y Appl. Phys. Lett. 931831046 (2008)

[5] Mano T. and Koguchi N. J. Cryst. Growth 278, 108 (2005)

[6] Mano T., Kuroda T., Sanguinetti S., Ochiai T., Tateno T., Kim J., Noda T., Kawabe M., Sakoda K., Kido G. and Koguchi N. Nano Letters 5(3), 425 (2005)

[7] Kuroda T., Mano T., Ochiai T., Sanguinetti S., Noda T., Kuroda K., Sakoda K., Kido G. and Koguchi N. Physica E 32, 46 (2006)

[8]Wang Zh. M., Liang B. L., Sablon K. A. and Salamo G. J. Appl. Phys. Lett. 90113120 (2007)

[9] Kiravittaya S, Rastelli A, and Schmidt O. G, Rep. Prog. Phys. 72, 046502 (2009)

[10] Imamoglu A, Awschalom D.D, Burkard G, DiVincenzo D. P, Loss D, Sherwin M, and Small A, Phys. Rev. Lett. 83, 4204 (1999)

[11] Hennessy K, Badolato A, Wigner M, Gerace D, Atatüre M, Gulde S, Fält D, Hu E.L, and Imamoglu A, Nature 445896 (2007)

[12] Bracker A.S, Scheibner M, Doty M. F, Stinaff E.A, Ponomarev I.V, Kim J. C, Whitman L. J, Reinecke T. L, and Gammon D, Appl. Phys. Lett. 89233110 (2006) 
[13] Molina S I, Ben T, Sales D L, Pizarro J, Galindo P L, Varela M, Pennycook S J, Fuster D, González Y and González L Nanotechnology 17 5652-5658 (2006)

[14] Sales D L, J Pizarro, Galindo P L, García R, Trevise G, Frigeri P, Nasi L, Franchi S and Molina S I Nanotechnology 18475503 (2007)

[15] Alonso-González P, Doctoral Thesis (2009)

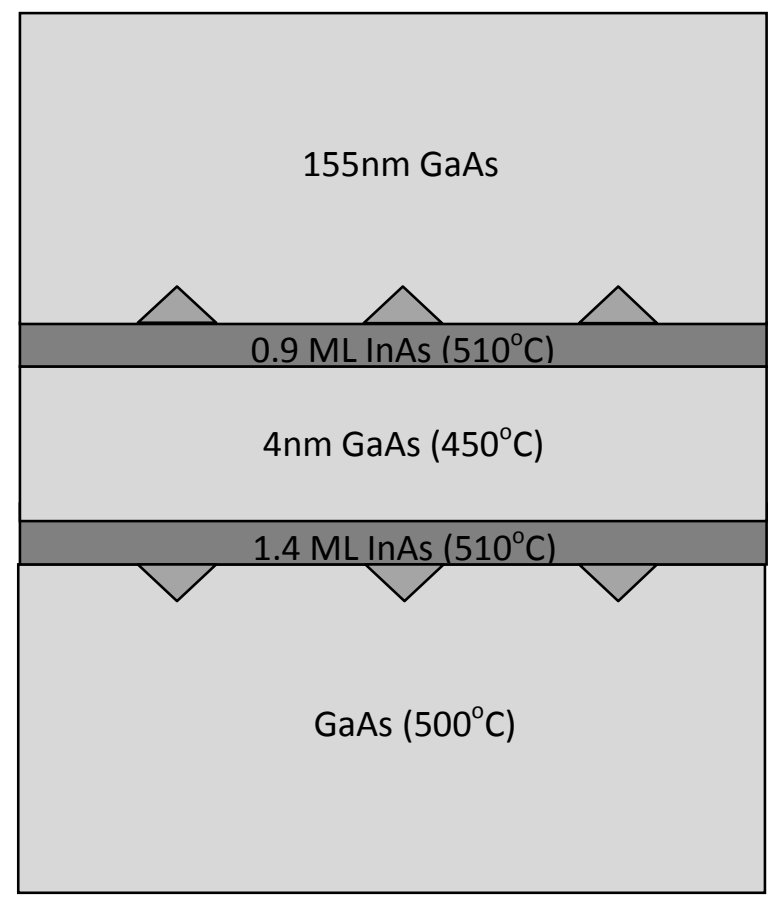

Fig.1. Schematic diagram of InAs/GaAs QDM structures. Thickness and growth temperature used for each layer are shown. 


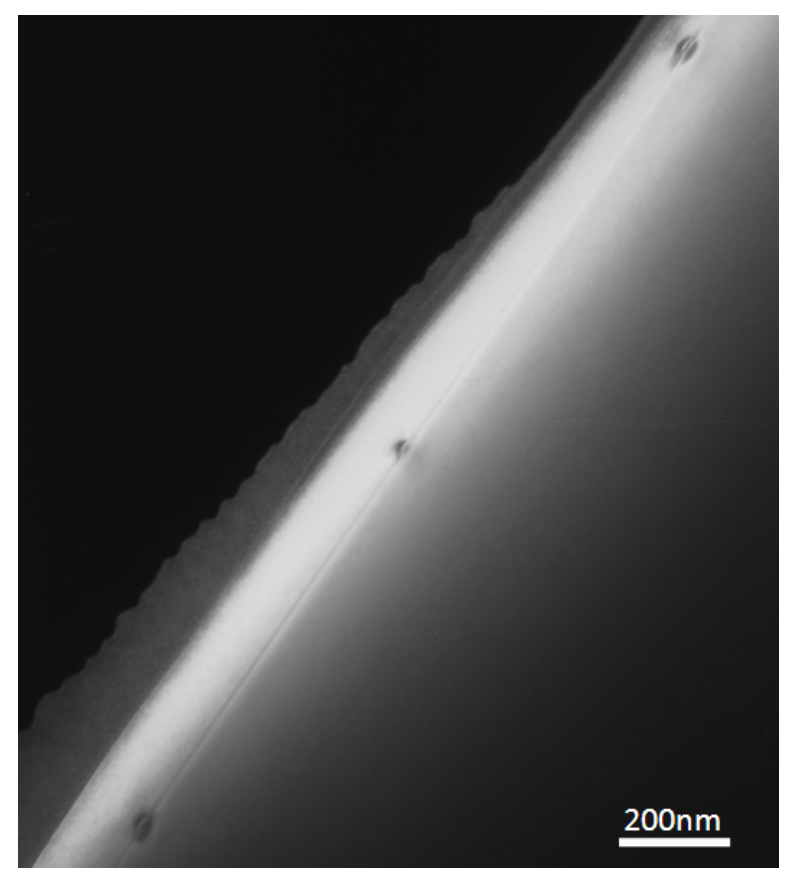

Fig. 2. Cross-sectional 002 DF TEM image.

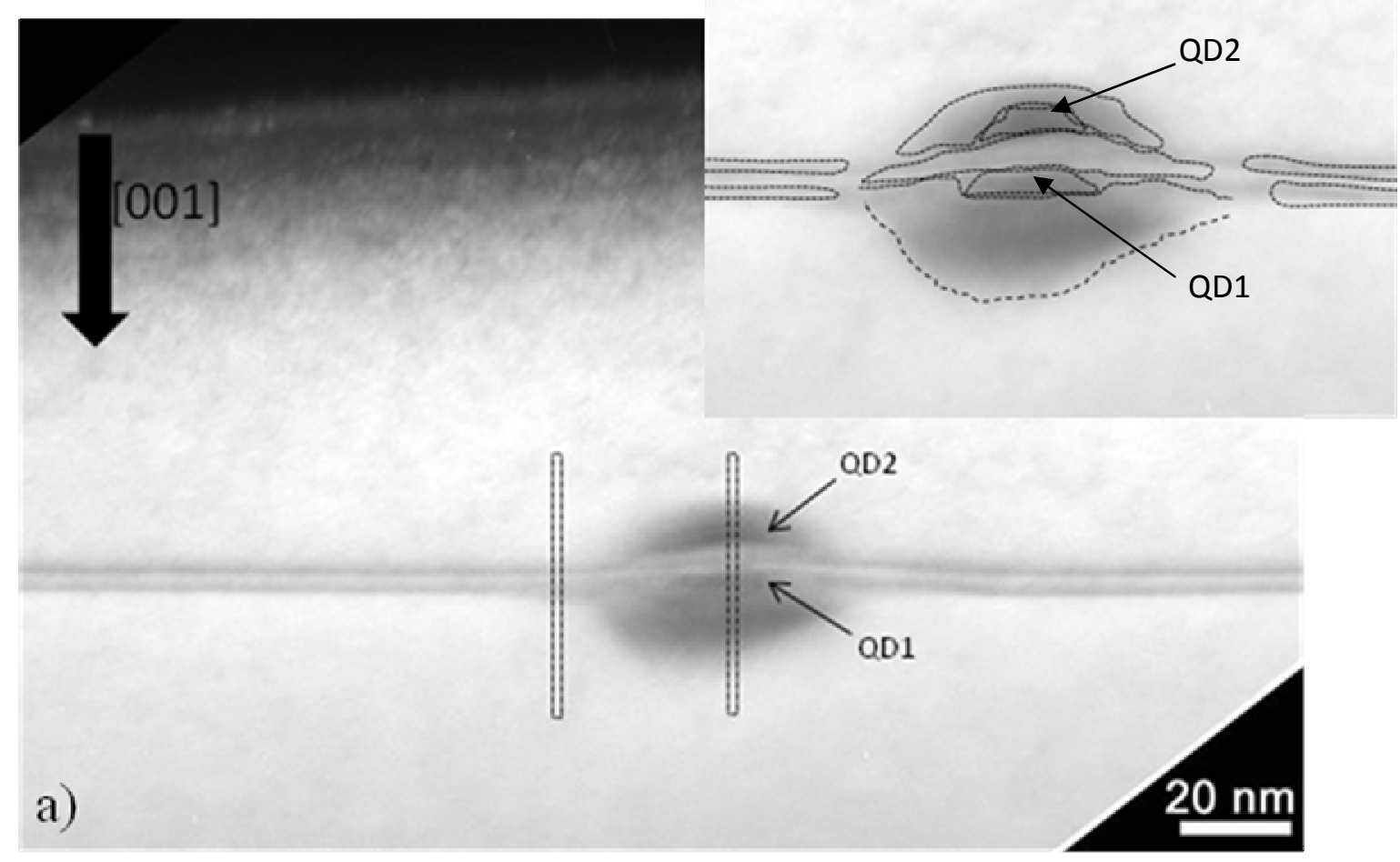



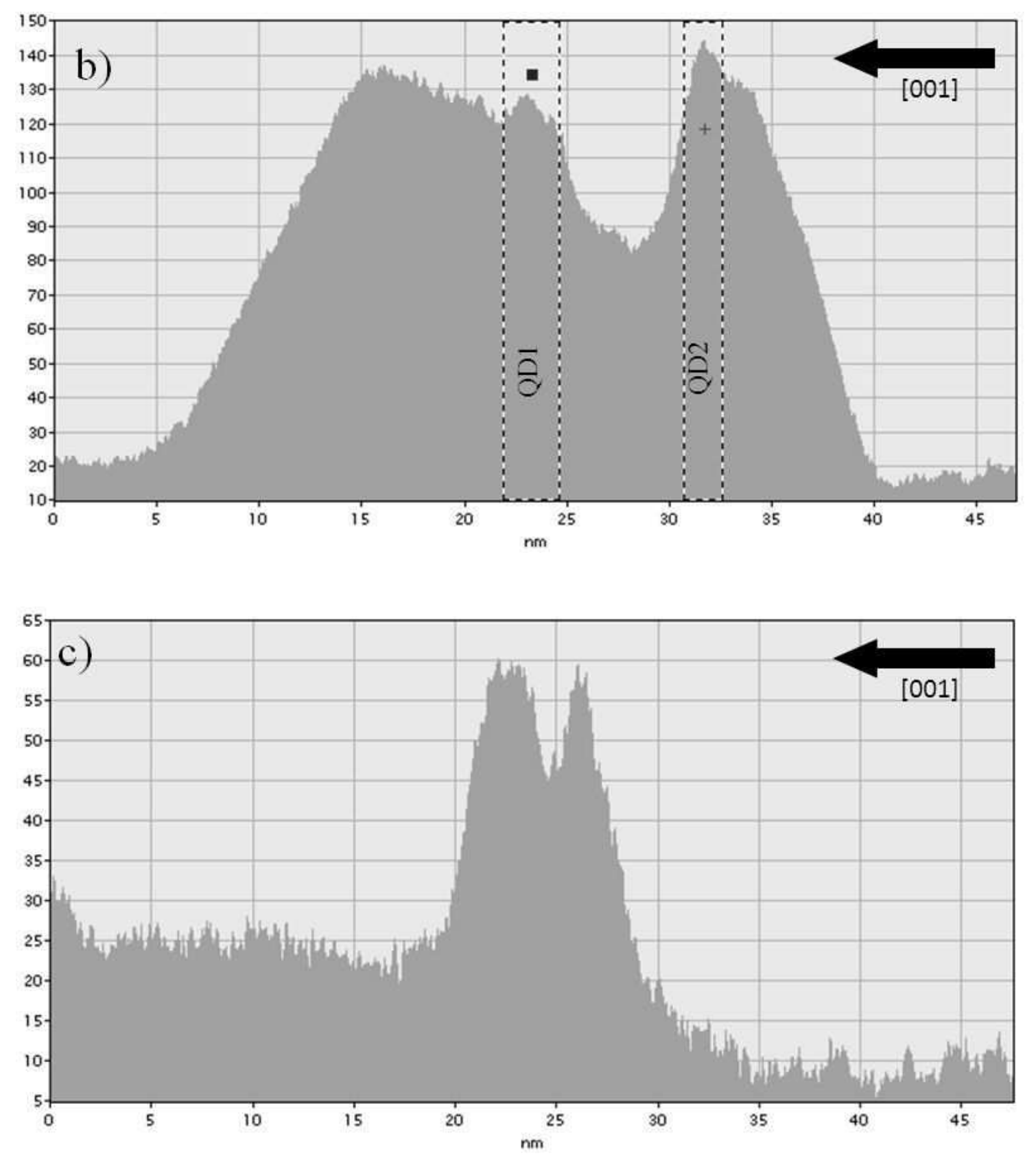

Fig. 3. (a) A quantum dots molecule and wetting layers in detail (002) DF image. (b) Intensity profile across a quantum dots molecule. (c) Intensity profile across the wetting layers close to the QDM nanostructure. Profiles have been taken from the negative image on fig. 3(a) to improve the observation of the peaks. 